\title{
KINETIC STUdy OF CHROMIUM (III) - PVP COMPLEX BY USING RADIO-TRACER TECHNIQUE
}

\author{
Ayan Das ${ }^{1}$ Aniruddha Roy ${ }^{2}$ and Nirmal Paul ${ }^{3}$ \\ 1,2,3 Department of Applied Science \& Humanities , Global Institute of \\ Management \& Technology, Krishnagar, Nadia, West Bengal, India \\ ${ }^{1}$ das.ayan84@gmail.com, ${ }^{2}$ aniruddha.rick@yahoo.com, \\ ${ }^{3}$ paul.nirmal7@gmail.com
}

\begin{abstract}
Complexes of metal ions with organic ligands play an important role in many fields of our life. These metal complexes are widely used in medical diagnosis and therapeutic analysis. These complexes are very familiar with radioisotopes exerting precise chemical and physical properties. Generally, chromium is considered as toxic and essential element depending on its oxidation state in the biological system. Here the dynamic dissociation constants of the complex of Chromium (III) with excellent biocompatible polymer PVP (poly-N-vinylpyrolidone) have been determined. The dissociation constants will give us the idea about the stability of the said complexes. As PVP is a good ligand with different metals within a wide range of $p H$. Ingestion techniques of radioisotopes to the biological systems may be nicely polished with the knowledge of this kinetic study with chromium. Green methods have been carried out during the whole period of the experiments to maintain the environmental friendly aspects.
\end{abstract}

\section{KEYWORDS}

HPGe detector, PVP (Poly- $N$-vinylpyrrolidone), radiotracer, $\mathrm{pH}$, dialysis sac, radionuclide, (n, $\Upsilon$ ) reaction, multichannel analyser, chromium (III).

\section{INTRODUCTION}

Worldwide demand for environmental friendly chemical processes and products requires the development of novel and cost-effective approaches to pollution prevention. One of the most attractive concepts for pollution prevention is green chemistry, which is best defined as the utilization of a set of principles that reduces or eliminates the use or generation of hazardous substances in the design, manufacture and applications of chemical products (Anastas and Warner, 1998[1]). To keep our method clean, deliberately no other chemical than PVP has been used for this study.

Complexes of metal ions with organic ligands play an important role in many different disciplines. For examples in environmental chemistry where investigations are carried out about which metals are complexed by humic and fulvic acid (van den Bergh et.al. 2001[2]; Sekaly et.al. 2003[3]; Procopio et.al. 1997[4]) or in radiochemistry, where a topic is to find suitable ligands for radio-nuclides relevant for applications in medical diagnosis and therapy. The precise chemical and physical properties of these metal complexes are rarely known, since the radioisotopes used are ideally nuclides of high specific activity and therefore small mass, which complicates handling of weighable amounts and rules out the exact characterization of the compounds. When

Rupak Bhattacharyya et al. (Eds) : ACER 2013,

pp. 461-471, 2013. (C) CS \& IT-CSCP 2013

DOI : $10.5121 /$ csit.2013.3243 
these compounds are used in nuclear medicine of radio-pharmacy it is indispensable that the metal complexes stay intact (Li et.al. 2000[5]) during transport to the desired location, which means a they should neither be metabolized nor should the complexed metal ion get lost because of insufficient stability of the metal complex.

Chromium is considered both as toxic and essential element depending on its oxidation state. For example, $\mathrm{Cr}$ (III) is an essential nutrient element while $\mathrm{Cr}$ (VI) is a carcinogen. For excellent biocompatibility and chemical inertness of poly-N-vinylpyrrolidone (PVP), it has got extensive application in drug formulation as drug base or excipient in our daily life. Therefore it is of utmost importance to know the binding ability of PVP with different trace metals present in human body. Determination of kinetics of radionuclide complexes is an important step in modern day radiopharmaceutical research. It is indispensable that the metal complexes should not get lost because of insufficient stability. At the same time it is also important that the metal complex should release the desired radionuclide after reaching the specific site in the body. These two aspects can be handled when an idea about their relative dissociation is known and as per the requirement a specific ligand can be chosen for a particular metal radionuclide. Recently, Lahiri and Sarkar (2007),[6,7] proposed an excellent and simple modification of FISRE technique (Wierczinski et.al. 2006[8]) by which dynamic dissociation constant of metal complexed with bulky ligand can easily be determined.

These facts prompted us to investigate the complexation of PVP towards Cr (III) labelled with

${ }^{51} \mathrm{Cr}$ radio-nuclides with the aid of dialysis technique. In dialysis technique it is expected that the polymer and metal-polymer complex would remain inside the bag due to the polymer's huge molecular weight $(40 \mathrm{kDa})$, and only the free ions would come out of the bag. The stability of the complex has been examined by measuring the dissociation constant $\left(\mathrm{k}_{\mathrm{d}}\right)$ from the slope of the plot of time vs. $\ln$ [Cr (III)-PVP].

The later on sections will provide the short overview of the related topics, experimental results, its discussions, validation of the findings and conclusive measure to this work.

\section{AN OVERVIEW OF SOME RELATED TOPICS}

In this section we have discussed some necessary topics used in the construction of the article. In subsections 2.1, 2.2, 2.3, 2.4, 2.5, 2.6, we have discussed on Chromium, radio-tracer, detection technique, PVP and Cr (III)-PVP complex respectively.

\subsection{Chromium at a glance}

Element chromium was discovered in crocoites ( $\mathrm{PbCrO} 4)$ by Vaquelin in 1798(Braceloux, 1999[9]). Carcinogenic effects of hexa-valent chromium were discovered towards the end of the $19^{\text {th }}$ century, when nose tumours in workers handling chromium pigments in Scotland were described (Cohen et.al.,1993[10]).Then many cases of carcinogenic effects of hexavalent chromium were found by the researchers. But trivalent chromium is very essential nutrient to human body which helps in metabolism of carbohydrates, proteins and lipids. Although chromium may theoretically be found in all oxidation state from -2 to +6 , it is most often found in $0,+2,+3$ and +6 . Elemental chromium ( 0 ) is not naturally present in earth crust and biologically inert in character. Almost all naturally occurring chromium is trivalent while hexavalent chromium is mostly of industrial origin. Most of the chromium compounds are halides, oxides or sulphides. Chromium has four naturally occurring isotopes, viz., ${ }^{50} \mathrm{Cr}(4.345 \%),{ }^{52} \mathrm{Cr}(83.789 \%)$, ${ }^{53} \mathrm{Cr}(9.501 \%)$, and ${ }^{54} \mathrm{Cr}(2.365 \%)$. It has a metallic Body Centred Cubic structures. Chromium is very toxic in large doses but in small doses it is an essential nutrient. Trivalent chromium plays a significant role in metabolism of cholesterol, lipids, carbohydrates, etc. (Anderson, 1986[11]; 
Versieck and Cornelis, 1989[12]). The radioisotopes of chromium, ${ }^{51} \mathrm{Cr}$ (T1/2 =27.7 days), is used to determine total blood cell volume and gastro-intestinal protein loss. In medicine, chromium compounds are used in astringents and antiseptics.

\subsection{Radiotracer}

George De Hevesy introduced the radiotracer technique by which radioisotopes could be used to investigate the behaviour of stable atoms. This technique is second to none in its analytical power. Radiotracer is a radioactive isotope of an element whose behaviour is identical to that of the same element. In this technique, the substances labelled with radioisotopes in very small amount(trace) allows one to study a number of physical and biological systems(without perturbing the system).This technique is used in very wide area from chemistry to biology, ecology to material science, medical to engineering sciences and many more.

The most important assumption made in the use of radiotracers is that the radioactive material will blend in perfectly with the system under study. The radioactivity or radiation emitted by the radiotracers will not adversely affect any components of the system and the tracer will behave in a way that is indistinguishable from the non-radioactive materials except for the emitted radiation (Ehmann et.al., 1991[13]).There are several factors on which this technique depends, viz.,half-life time of the radioisotopes, nature of the radiation emitted by the radioisotopes, energy of the radiation,etc. The chief criterion for the selection of tracer is that the tracer does not change physical, chemical and metabolic properties of the experimental system. Above all this technique specifically depends on the particular application.

\subsection{Production of radiotracer}

With the success of atomic pile in1942 for the first time, was available for isotope production, with the neutron as a bombarding particle. Nuclear reactor is a chief source of neutrons, which provides fluxes of thermal and fast neutrons of the order of $10^{12}-10^{14} \mathrm{n} / \mathrm{cm}^{2} / \mathrm{sec}$. In most of the cases radiotracers are produced through $(n, \Upsilon)$ reactions using thermal on neutrons. The high thermal neutron absorption cross-section (the probability of occurring a nuclear reaction) for most of the elements made the slow neutrons as most useful projectiles. However, the radio nuclides produced by neutron absorption of low specific activity. Isotopes of all the elements can't be produced through thermal neutron activation. There many other procedures to get the radio tracers like charged particle activation with proton or alpha particles as projectiles etc. But, the neutron activation is very easy and significant tool in this purpose.

In $(\mathrm{n}, \Upsilon)$ reaction, the target nucleus first absorbs a neutron and then spontaneously emits either a $\Upsilon$ photon or a charged particle. ${ }^{59}$ Co $(n, \Upsilon){ }^{60}$ Co $\left(t_{112}=5.3\right.$ years is an examples of $(n, \Upsilon)$ reactions.

\subsection{Detection of radioactivity}

Nuclear radiation detectors require for identification and characterisation of different radiation and assay of radioactivity in the study of radioactivity in the studies of nuclear reactions, nuclear spectroscopy and radio chemical separations. All methods for detection of radioactivity are based on interaction of charged particles or electromagnetic radiations with matter; which is to produced electron in matter and detected by obtaining an electric charged pulses, this electric pulse is directly proportional of the incident radiations. Ion chamber proportional counter, G.M. counter, Scintillation detectors are the examples of radioactivity detectors. But HPGe detector (High Purity Germanium Detector) is used in this case as it is more convenient and widely used to detect $\Upsilon \mathrm{r}$ radiations. 
In the latter on sub-sections, the details of HPGe detector has been discussed briefly.

\subsubsection{HPGe Detector}

This is a semi-conductor detector is made of crystalline semi conductor material germanium in highly pure state. The band gap energy is $0.7 \mathrm{eV}$ in germanium. The passage of radiation may inject the enough energy to the system to raise an electron from the valance band to conduction band, thus creating an electron-hole pair. Both electron and the hole may migrate through the crystal in the applied electric field and produce an electrical that marks the passage of the radiation. For $\Upsilon$-ray detection, highly pure germanium is preferred because of its higher atomic number. Germanium detector is operated at low temperature because of smaller bent gap of germanium. The high purity germanium crystal is made of single crystal of the element with an impurity concentration less than $3 \times 10^{-10} / \mathrm{cm}^{3}$.

The detector is always cooled by liquid nitrogen to avoid the problem due to thermal excitation of electron always exist. The principle of these detectors is governed by $\Upsilon$-ray spectroscopy with the highest resolution of $\Upsilon$ - ray energies from a few KeVs to $10 \mathrm{MeVs}$. The parameter of the detector is efficiency and resolution.

Efficiency $=$ number of pulses recorded /no of radiations emitted by the source.

Resolutions=full width at half maximum/centroid of the peak energy.

\section{5. poly-(N-vinylpyrrolidone)}

It is a bio-compatible as well as biodegradable polymer. The three basic criteria of the polymer are - a. It should be either biodegradable or inert to human body, b. It should not evoke any unwanted reactions inside the body and c. It should be eliminated from the body as soon as possible after the targeted delivery. PVP is a synthetic homo polymer and can be readily prepared from its monomer $\mathrm{n}$ vinyl pyrrolidone. Due to its excellent biocompatibility and chemical inertness, it has got extensive application in drug formulation and other clinical and biological fields. This polymer has a wide range of solubility in a large number of organic solvent. As it is highly soluble in water, it can be utilised in green chemistry processes. PVP binds excellently to polar molecules. Recently, it is widely used in pharmaceutical industries as filler, in personal care products etc.PVP is of utmost important in our everyday life. Therefore, it is very crucial and important to know the binding ability of PVP to different tress metals present in human body.

\subsection{PVP-metal complexation: A brief overview}

The analytical chemistry of the polymer PVP was studied by Tokman et.al, 2004[14]. PVP forms complexes with metals via oxygen and nitrogen donor sites, due to the high polarity of the lactum ring conferred by resonance stabilisation facilitated by a near planer geometry. The interaction between PVP and some metal ions was also investigated by Liu et.al, 2000[5]. They studied the complexation of the metal cations like $\mathrm{Fe}^{3+}, \mathrm{Co}^{2+}$ and $\mathrm{Ni}^{2+}$ with PVP in ethanol and water and reported the formation of complexes with the help of UV-Vis and IR spectroscopy. From the findings of the others it is cleared that the metal binding areas of PVP and co-polymers of PVp were the carbonyl oxygen's and the nitrogen's of the polymer in aqueous solution. Again, Maturana et.al.[15],2000 successfully used PVP in kerosene-octanol mixture as liquid-liquid extract ant for the metals $\mathrm{Cu}^{2+}, \mathrm{Zn}^{2+}, \mathrm{Co}^{2+}, \mathrm{Ni}^{2+}, \mathrm{Cd}^{2+}, \mathrm{Pb}^{2+}, \mathrm{Hg}^{2+}, \mathrm{Cr}^{3+}$ and $\mathrm{Fe}^{3+}$ and determine the interactions as a function of $\mathrm{pH}$ as well as kinetic characteristics. Diaz et.al, 2004[16] studied the synthesis and characterisation of different PVP-Co and PVP-Cu complexes in solid states. 
Despite of the suitable physical, chemical land biochemical properties, a limited amount of information is available concerning the complexation ability of PVP of with radionuclides. The tremendous significance of ${ }^{66,67} \mathrm{Ga}$ and ${ }^{199} \mathrm{Tl}$ radionuclide's in nuclear medicine, the possibility of formation of PVP complexes with above mentioned radionuclide's has been examined. It has been found that 5\% aqueous solution of PVP can almost quantitatively back extract those radionuclide's from organic phase, which can prove the complexation ability of PVP. TI PVP and Ga PVP complexes have been studied by Lahiri and Sarkar, 2007[6, 7]. It has been found that in acidic $\mathrm{pH}$ Ga has a high tendency for the formation of Ga PVP complex, while the same is true for $\mathrm{Cu}$ and $\mathrm{Zn}$ in neutral and basic $\mathrm{pH}$. Ge is merely inert towards the interaction with PVP at all pH values, sarkar et.al. 2007[6, 7]. These observation develops a good separation techniques involving dialysis of different PVP complexes with radionuclides against triple distilled water. This method is applicable for tress scale to macro scale with very high separation factors, Lahiri and Sarkar, 2007[6, 7].

\section{EXPERIMENTAL}

Here, we will discuss about all the procedures which have been followed during the whole period of our investigations. Specifically all the work has been illustrated in different classified subsections as follows.

\subsection{Production Of Radioisotopes and Chemical Reagents}

In this section production of radio-nuclides and other chemicals used have been discussed duly.

\subsubsection{Irradiation}

A weighed amount of chromium nitrate, $\mathrm{Cr}\left(\mathrm{NO}_{3}\right)_{3} \cdot 9 \mathrm{H}_{2} \mathrm{O}$ salt has been subjected to neutron irradiation in the CIRUS reactor,BARC,Mumbai with a neutron flux $1 \times 10^{12} \mathrm{n} . \mathrm{cm}^{-2} . \mathrm{s}^{-1}$. The nuclear reaction for the production of chromium radionuclide is ${ }^{50} \mathrm{Cr}(\mathrm{n}, \Upsilon)^{51} \mathrm{Cr}$. The half-life of ${ }^{51} \mathrm{Cr}$ is 27.7 days and the $\mathrm{Y}$-ray energy is $320.08 \mathrm{KeV}$. After irradiation the salt is dissolved in deionised water.

Polyvinylpyrrolidone (M.W. =40,000) was obtained from SRL, India. Dialysis sac (D606625EA) has been procured from SIGMA-ALDRICH. All other reagents are of analytical grade and used without further purification.

\subsubsection{Detection}

An HPGe detector of $2.13 \mathrm{KeV}$ resolutions at $1.33 \mathrm{MeV}$ in conjugation with $\mathrm{PC}$ based multichannel analyzer, has been used for $\Upsilon$-spectroscopic studies.

\subsection{Studies on the Complexation of PVP with ${ }^{51} \mathrm{Cr}$ (III)}

Here, the complexation of $\mathrm{Cr}$ (III) with varying concentration of PVP solutions has been discussed.

\subsubsection{VARIATION of Concentration of PVP}

PVP solutions of various strengths $(5 \%, 10 \%$, and 20\%) have been made by dissolving calculated amount of PVP in triple distilled water. Dialysis sacs are cut into suitable lengths according to various volumes of dialysis solutions. The bag has been tied on one end with a cotton thread and 
checked for any leakage by triple distilled water. Dialysis has been carried out against triple distilled water with the help of magnetic stirrer at room temperature of $22^{\circ} \mathrm{C}$ to $23^{\circ} \mathrm{C}$. During dialysis every time water level has been maintained to dip the area of the bag containing the liquid.

Chromium $\left({ }^{51} \mathrm{Cr}\right)$ has been monitored by measuring the area of characteristic photo peak 320.08 $\mathrm{keV}$. The chromium salt has been dialyzed against triple distilled water. After definite time interval the counts of ${ }^{51} \mathrm{Cr}$ in the dialysis sacs are taken and compared with initial count of the sacs.

\subsection{2 pH Variation}

Studies on the complexation of PVP with Cr (III) are carried out by varying PVP concentration keeping $\mathrm{pH}$ fixed. Once the optimum PVP concentration has been observed then complexation is studied by varying $\mathrm{pH}$. All the sets have had $4 \mathrm{ml}$ of PVP of different concentrations and $1 \mathrm{ml}$ of ${ }^{51} \mathrm{Cr}$ (III) solution of different $\mathrm{pHs}$.Appropiate decay correction for ${ }^{51} \mathrm{Cr}$ has also been done for all experiments.

\section{RESULTS AND DISCUSSIONS}

In this section we will deal with the experimental results and make constructive discussions on them to get the actual picture. All matter has been discussed in different sub-sections.

\subsection{Experiment with Trace Scale ${ }^{51} \mathrm{Cr}$ (III)}

In order to get an idea about the stability of Cr (III) and PVP complex in trace scale, the retention of ${ }^{51} \mathrm{Cr}$ in the dialysis sac has been checked after definite time interval. Assuming first order reaction between PVP and ${ }^{51} \mathrm{Cr}$ radionuclide the dissociation rate of PVP-chromium radionuclide complex will be:

$$
-d\left[(M-L)^{m+n}\right] / d t=k_{d} \cdot\left[(M-L)^{m+n}\right]-k_{a}\left[M^{m}\right] \cdot\left[L^{n}\right] .
$$

Here, $\mathrm{M}$ represents $\mathrm{Cr}$ radionuclide and $\mathrm{L}$ represents PVP. The determination of dissociation constant may be evaluated with the help of FISRE (Free Ion Selective Radiotracer Extraction) technique (Wierczzinski et.al, [8]; Doornmaln et.al, 2000[17]). Due to the high surplus of inactive metal ions, the probability of 're-association' with radioactive metal ion can be neglected. During dialysis of the PVP-metal complexes, the dissociated nuclides are continuously removed from the dialysis sac. Therefore, it may be assumed that the 're-association' does not take place. Then the above equation may be simplified as follows:

$$
d\left[(M-L)^{m+n}\right] / d t=-k_{d} \cdot\left[(M-L)^{m+n}\right]
$$

The solution of the differential equation is:

$$
\left[\left({ }^{r a d} M-L\right){ }_{t}^{m+n}\right]=\left[\left({ }^{r a d} M-L\right)_{t o}{ }^{m+n}\right] \cdot e^{-k d . t}
$$

A plot of time vs. $\ln$ [Cr (III)-PVP] will give an idea about the dissociation constant ( $\left.\mathrm{k}_{\mathrm{d}}\right)$ by measuring the slope of the plot.

Figure 1 and 2 and 3 show the $\ln [\mathrm{Cr}(\mathrm{III})-\mathrm{PVP}]$ association in the dialysis sac with respect to time at $\mathrm{pH} 5$ with $5 \%, 10 \%$ and $20 \%$ PVP concentrations respectively. The $\mathrm{k}_{\mathrm{d}}$ value of Cr (III)-PVP complex at different concentrations of PVP reflects that the complex is more stable with $10 \%$ $\mathrm{PVP}$ at $\mathrm{pH} 5$. 


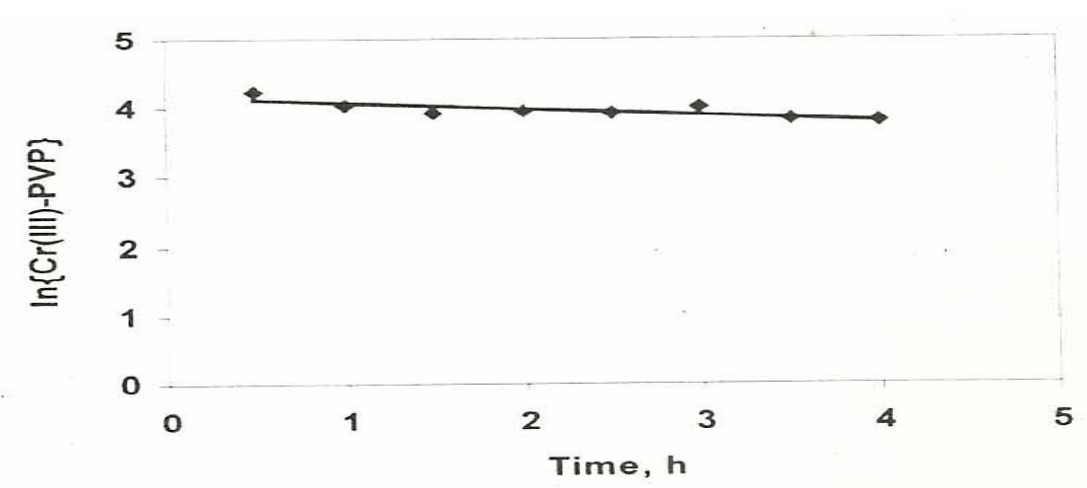

Figure 1. Dissociation of ${ }^{51} \mathrm{Cr}$ (III)-PVP complex with respect to time when dialyzed against triple distilled water with $5 \% \mathrm{PVP}$ at $\mathrm{pH} 5$

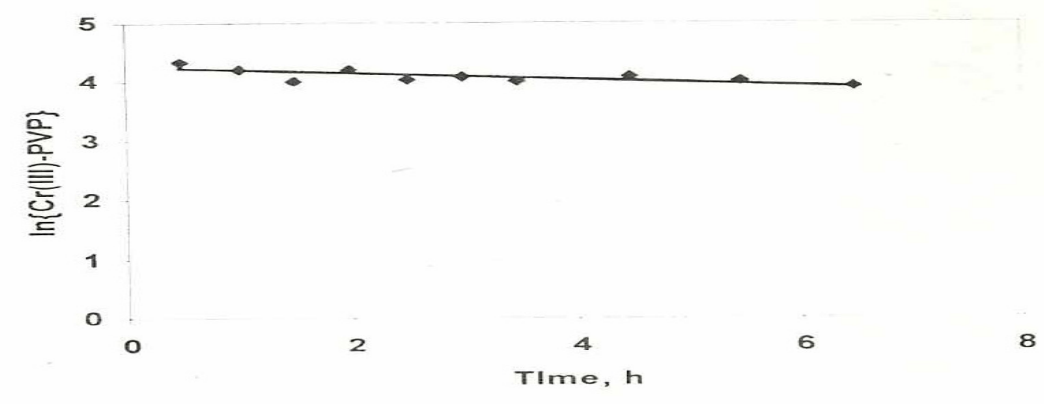

Figure 2. Dissociation of ${ }^{51} \mathrm{Cr}$ (III)-PVP complex with respect to time when dialyzed against tripled distilled water with $10 \% \mathrm{PVP}$ at $\mathrm{pH} 5$

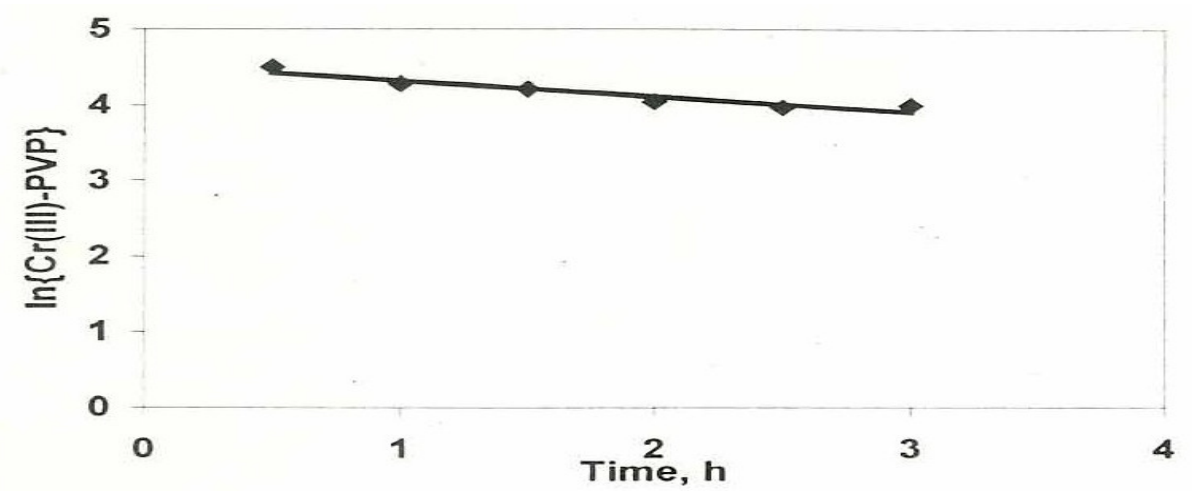

Figure 3. Dissociation of ${ }^{51} \mathrm{Cr}$ (III)-PVP complex with respect to time when dialyzed against triple distilled water with $20 \% \mathrm{PVP}$ at $\mathrm{pH} 5$

The dissociation constants determined may however slightly differ from their true value as the reassociation is completely ignored. In order to get an idea about the best $\mathrm{pH}$ for complexation of $\mathrm{Cr}$ (III) with PVP, the $\mathrm{pH}$ of the radio-nuclides plus PVP mixtures are varied keeping the concentration of PVP fixed at $10 \%$. 


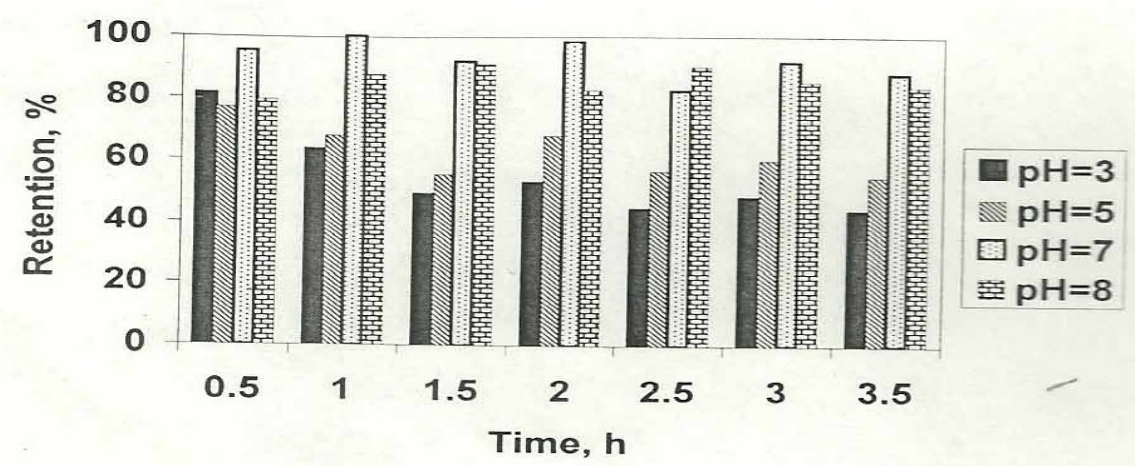

Figure 4. Retention percentage of ${ }^{51} \mathrm{Cr}$ (III)-PVP complex as a function of time with varying pHs keeping PVP concentration fixed at $10 \%$

From the experiment it has been found that at $10 \%$ PVP concentration the retention percentage of ${ }^{51} \mathrm{Cr}$ (III)-PVP complex in the dialysis sac increases in the following order: $\mathrm{pH} 3<\mathrm{pH} 5<\mathrm{pH} 8<\mathrm{pH} 7$ after 3.5 hours of dialysis (Figure 4). At the experimental condition about $88 \%$ retention of $\mathrm{Cr}$ (III)-PVP complex has been observed at $\mathrm{pH}$ 7.Again $76 \%$ of ${ }^{51} \mathrm{Cr}$ (III)-PVP complex retained in the dialysis sac even after 24 hours of dialysis. Thus, it can be concluded that PVP is an efficient reagent for binding of $\mathrm{Cr}$ (III) and therefore may be used for the removal of $\mathrm{Cr}$ (III).

The dissociation constant values $\left(\mathrm{k}_{\mathrm{d}}\right)$ of $\mathrm{Cr}$ (III)-PVP complexes at $\mathrm{pH}$ 3, 7 and 8 have also been calculated from their respective $\ln [\mathrm{Cr}(\mathrm{III})-\mathrm{PVP}]$ vs. time plots (Figure 5, 6 and 7). Figures 2, 4-6 show that $\mathrm{Cr}(\mathrm{III})-\mathrm{PVP}$ complexation is best at $\mathrm{pH} 7$ and $10 \%$ PVP concentration with $\mathrm{k}_{\mathrm{d}}=0.0377$ $\mathrm{h}^{-1}$.

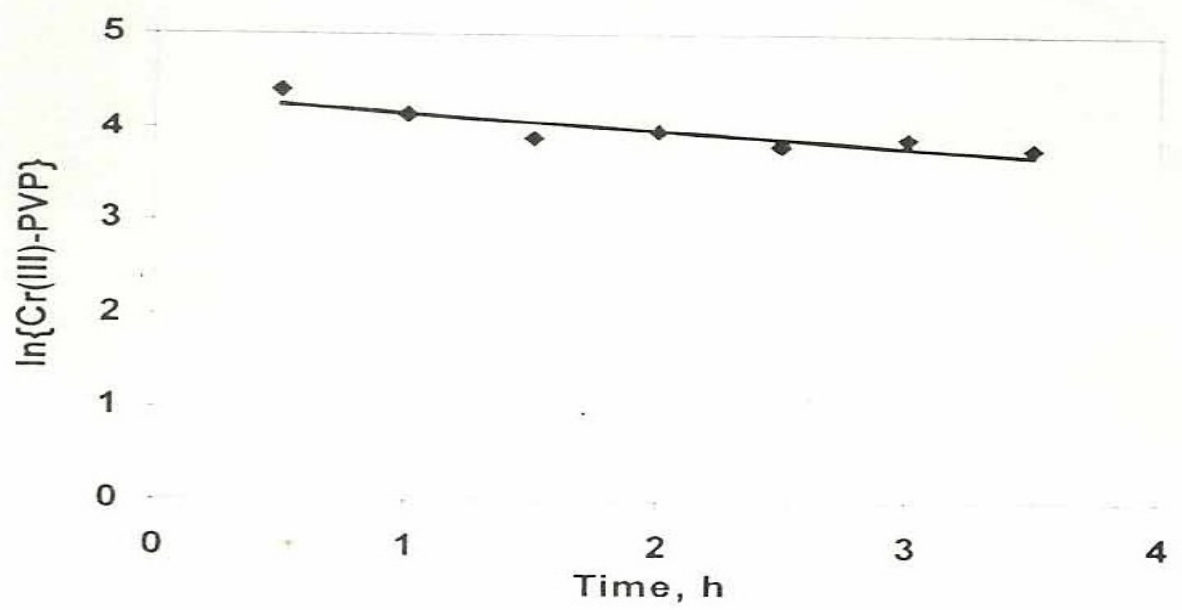

Figure 5. Dissociation of ${ }^{51} \mathrm{Cr}$ (III)-PVP complex with respect to tie when dialyzed against triple distilled water with $10 \% \mathrm{PVP}$ at $\mathrm{pH} 3$ 


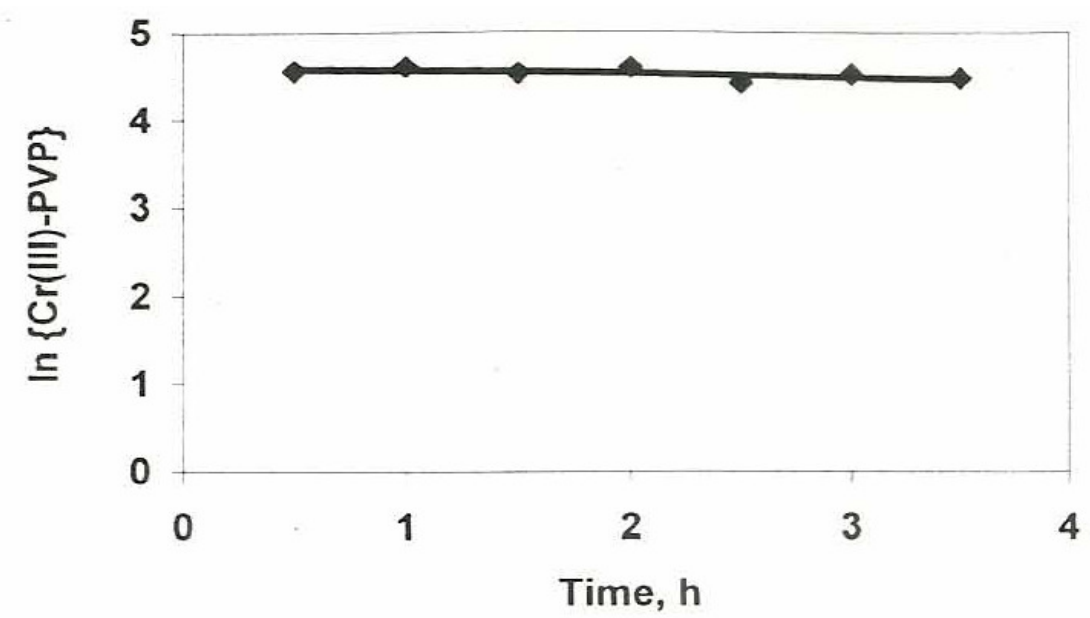

Figure 6. Dissociation of ${ }^{51} \mathrm{Cr}$ (III)-PVP complex with respect time when dialyzed against triple distilled water with $10 \% \mathrm{PVP}$ at $\mathrm{pH} 7$

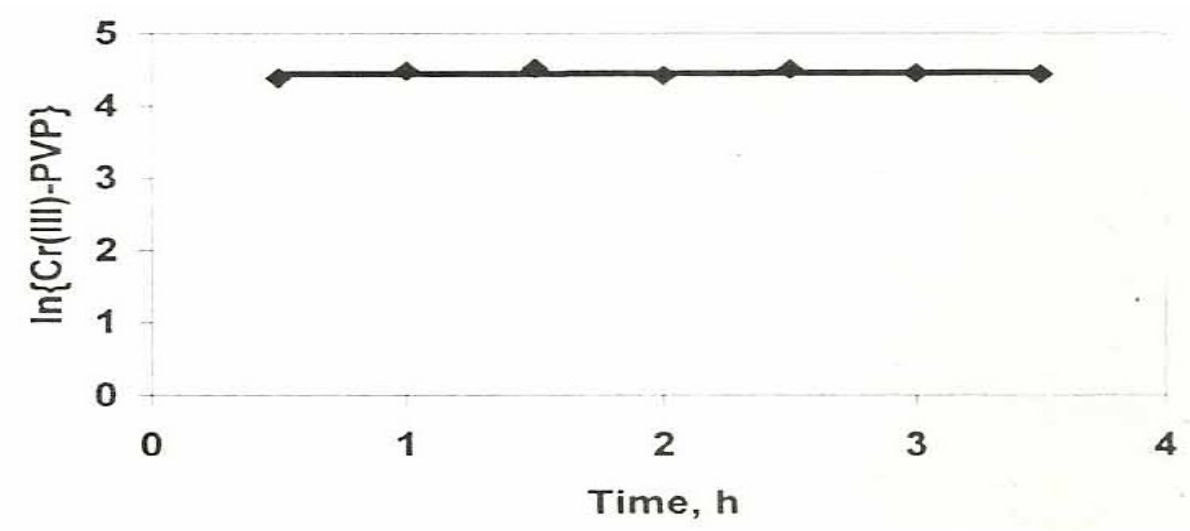

Figure 7. Dissociation of ${ }^{51} \mathrm{Cr}$ (III)-PVP complex with respect to time when dialyzed against triple distilled water with $10 \% \mathrm{PVP}$ at $\mathrm{pH} 8$

The dissociation constants $\left(\mathrm{k}_{\mathrm{d}}\right)$ of ${ }^{51} \mathrm{Cr}$ (III)-PVP complex with various concentration of PVP at pH 5 and $10 \%$ PVP at various pHs is represented in table 1 and table 2 respectively.

Table 1: Dissociation constants $\left(\mathrm{k}_{\mathrm{d}}\right)$ of ${ }^{51} \mathrm{Cr}$ (III)-PVP complex with various concentration of PVP at $\mathrm{pH} 5$

\begin{tabular}{|c|c|}
\hline PVP, $\%(\mathrm{w} / \mathrm{v})$ & Dissociation constant $\left(\mathrm{k}_{\mathrm{d}}\right) \mathrm{in}^{-1}$ \\
\hline 5 & 0.0907 \\
\hline 10 & 0.0507 \\
\hline 20 & 0.2034 \\
\hline
\end{tabular}

Table 2: Dissociation constants $\left(\mathrm{k}_{\mathrm{d}}\right)$ of ${ }^{51} \mathrm{Cr}$ (III)-PVP complex with $10 \% \mathrm{PVP}$ at various $\mathrm{pHs}$

\begin{tabular}{|c|c|}
\hline $\mathrm{pH}$ & Dissociation constant $\left(\mathrm{k}_{\mathrm{d}}\right)$ in $^{-1}$ \\
\hline 3 & 0.1782 \\
\hline 5 & 0.0507 \\
\hline 7 & 0.0377 \\
\hline
\end{tabular}



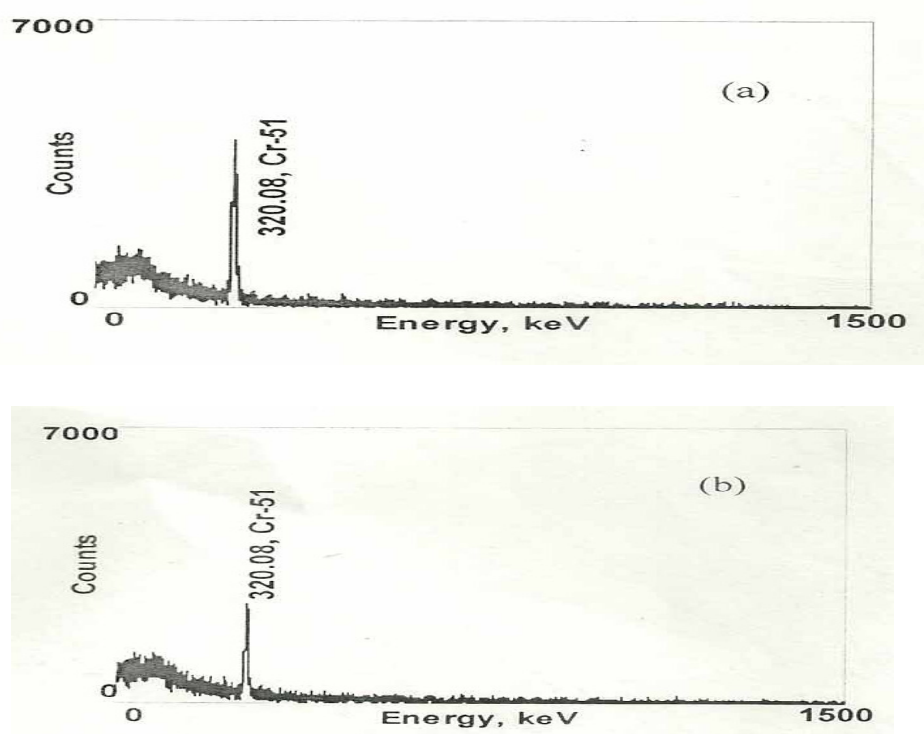

Figure 8. $\Upsilon$-ray spectra of ${ }^{51} \mathrm{Cr}$ (III)-PVP complex before dialysis (a) and after 3.5 hours of dialysis (b) with $10 \% \mathrm{PVP}$ at $\mathrm{pH} 7$

\section{CONCLUSIONS}

The dynamic dissociation constant of ${ }^{51} \mathrm{Cr}$ (III)-PVP complex has been determine at different concentration of PVP as well as at different pHs employing dialysis technique. Result shows that the complexation ability of PVP with ${ }^{51} \mathrm{Cr}(\mathrm{III})$ is more in neutral $\mathrm{pH}(\mathrm{pH} 7)$ rather than in acidic or alkaline pH.The dynamic dissociation constant of the ${ }^{51} \mathrm{Cr}(\mathrm{III})$-PVP complex is calculated as $0.0377 \mathrm{~h}^{-1}$ with $10 \% \mathrm{PVP}$ solution and $\mathrm{pH}$ 7. The present work preliminarily suggests about the possible use of $\mathrm{Cr}$ (III)-PVP complex as radiopharmaceutical. However, this work can be extended to study the stability of Cr (III)-PVP complex against human blood serum as well as bio-distribution of $\mathrm{Cr}$ (III)-PVP complex.

The experiment fulfils the agenda of green chemistry as water soluble PVP has been in all the dialysis processes against triple distilled water. Coupled to this, PVP is one of the most suitable polymers, frequently used as an inert binder of drug, completely compatible with the human body system and non-toxic. Again, Cr (III)-PVP complexes can be exploited in the field of biological studies. Further study is needed to show the stability of the Cr (III)-PVP complex during transportation and release of metal at the desired sites.

\section{ACKNOWLEDGEMENT}

We want to express our gratitude to SINP, Kolkata for their technical support all through the work. We want to thank all of our co-workers for their endless co-operation and are also grateful to our family for their support during the whole period of our work. We also want to thank all of our colleagues and to all who supported us in this regard especially Dr. Rupak Bhattacharyya. Finally, we sincerely thank the anonymous reviewers and the editors for their through and constructive suggestions to give proper shape to the manuscript. 


\section{REFERENCES}

[1] Anastas P., Warner J.(1998):Green Chemistry: Theory and Practice; Oxford University Press: Oxford.

[2] Bergh J. Van den, J Jakubawski B., Burba P. (2001): Investigation on the conditional kinetic and thermodynamics stability of aquatic humic substance- metal complexes by means of EDTA exchange, ultra filtration and atomic spectrometry. Talanta, 55, 587.

[3] Sekaly A. L. R., Murimboh J., Hassan N. M., Mandal R., Ben Younes M. E.,Chakrabarti C., Back M. H. (2003): Environmental Science \& Technology, 37, 66.

[4] Procopio J. R., Mar Ortiz Viana M. del, Hernandez L. H.,(1997): Environmental Science \& Technology, 31, 3081.

[5] Liu M., Yan X., Liu H., Yu W.(2000): An investigation of the interaction between polyvinylpyrrolidone and metal cations. Reactive and Functional Polymers, 44, 55.

[6] Lahiri S., Sarkar S., (2007): A green method of separation of iron and cobalt by dialysis of polyvinylpyrrolidonemetal complexes. Appl.Radiat.Isot.,65,309

[7] Lahiri S., Sarkar S., (2007): Studies on ${ }^{66,67} \mathrm{Ga}$ and ${ }^{199} \mathrm{Tl}-$ polyvinylpyrrolidone complexes. Appl.Radiat.Isot.,65,387

[8] Wierczinski B., Denkova A. G., Peters J.A., Wolterbeek H.T.,2006. Kinetics stability of metal complexesDetermination of $k_{a}$ and $k_{d}$ using radiotracers. In: Lahiri S., Nayak D., Mukhopadhyay A.(Eds), Application of radiotracers Chemical, Environmental and Biological Sciences. Saha Institute of Nuclear Physics, Kolkata, India, pp.112.

[9] Barceloux D. G. (1999): Chromium, Clinical Toxicology, 37, 173

[10] Cohen M.D., Kargacin B., Klein C.B., and Costa M. (1993): Mechanism of chromium carcinogenity and toxicity. Critical Reviews in Toxicology, 23, 255

[11] Anderson R.A. (1986): Chromium metabolism and its role in disease processes in man. Clin.Physiol.Biochem., 4, 31

[12] Versieck J., Cornelis R. (1989): Trace Element in Human Plasma or Serum, CRC Press, Boca Raton, FL.

[13] Ehmann W.D., Vance D.E., (1991): Radiochemistry and Nuclear Methods of Analysis, John Wiley \& Sons, New York.

[14] Tokman N., Akman S., Ozeroglu C. (2004): Determination of lead, copper and manganese by graphite furnace atomic absorption spectrometry after separation/concentration using a water-soluble polymer. Talanta, 63, 699.

[15] Maturana H.A., Peric I.M., Pooley S.A., Rivs B.L., (2000): Polyvinylpyrrolidone as metal ion liquid-liquid extractant. Polymer Bulletin, 45, 425

[16] Diaz E., Valenciano R.P., Katime I.A., (2004): Study of complexes of polyvinylpyrrolidone with copper and cobalt on solid state. Jrnl.Appl.Polymer Science., 93, 1512

[17] Doornmalen J. van,Elteren J.T. van Goeij J.J.M. de(2000): A Chromatographic Technique to Investigate the Lability of Copper Complexes under Steady-State Conditions Using High Specific Activity ${ }^{64} \mathrm{Cu}$.Anal.Chem., 72,3043

\section{AUTHORS}

Ayan Das has been working as an assistant professor in chemistry under the Department of Applied Science and Humanities in Global Institute of Management \& Technology, Krishnagar, Nadia, West Bengal. He possesses 5 years teaching experience at college level.

Aniruddha Roy has also been working as an assistant professor in chemistry under the Department of Applied Science and Humanities in Global Institute of Management \& Technology, Krishnagar, Nadia, West Bengal. He is skillful in mineral ore and metal analysis. He spent a long time of his career in different geo-chemical laboratories.

Nirmal Paul has been acting as a technical assistant in chemistry under the department of Applied Science and HUMANITIES in Global Institute of Management \& Technology, Krishnagar, Nadia, West Bengal.

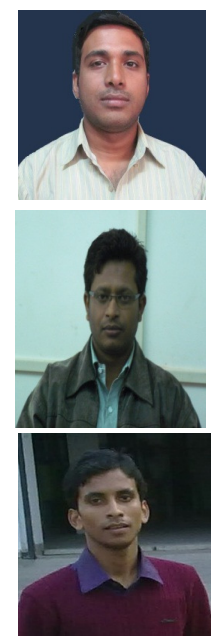

